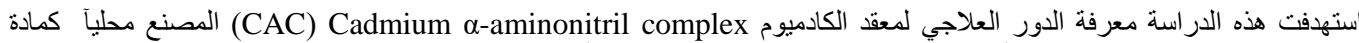

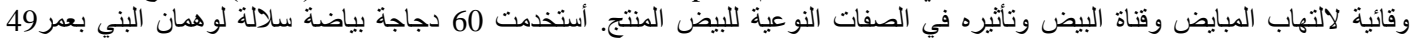

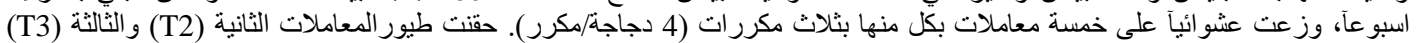

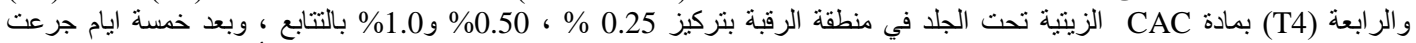

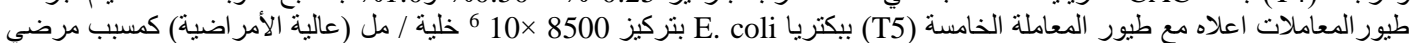

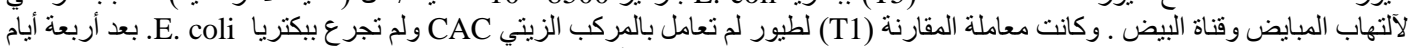

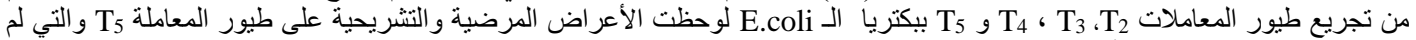

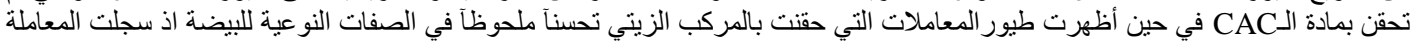

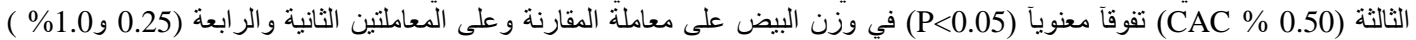

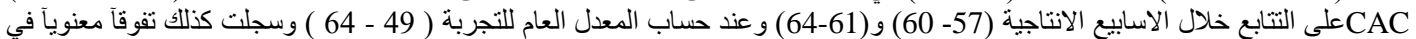

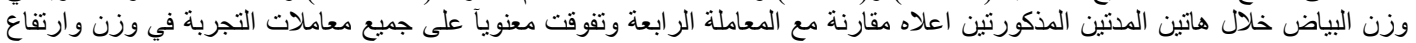

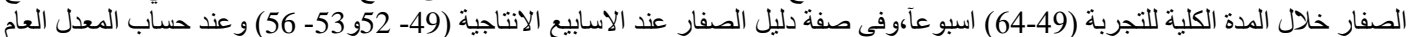

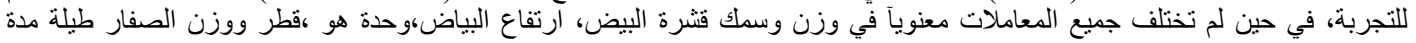

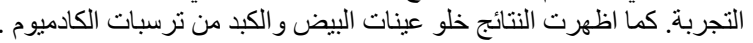

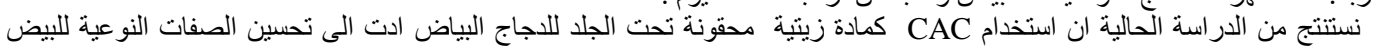

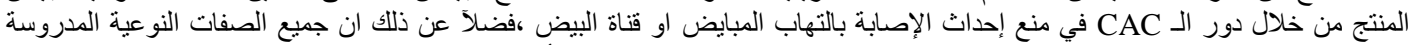

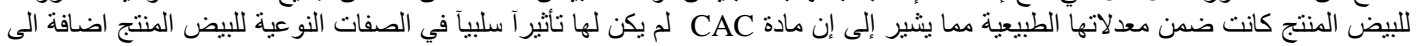

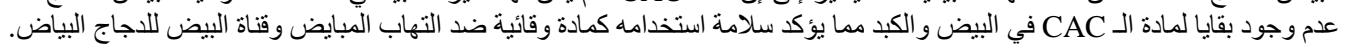
كلمات دالة : دجاج بيض المائدة، الصفات النوعية للبيض، معقد الكادميوم .

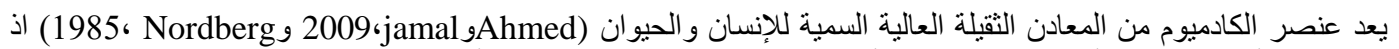

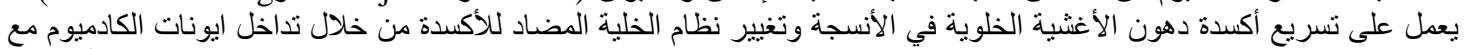

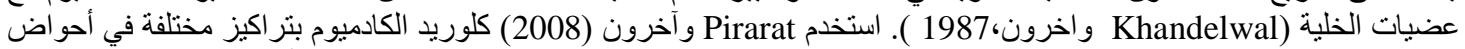

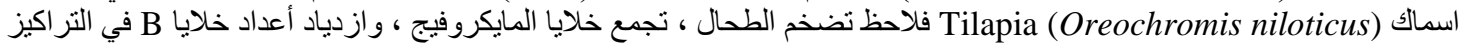

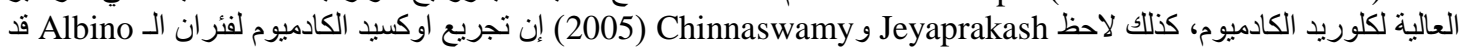

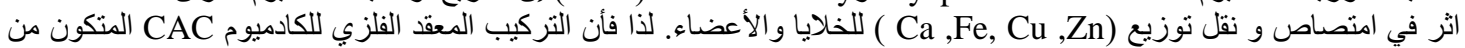

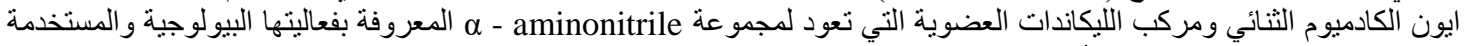

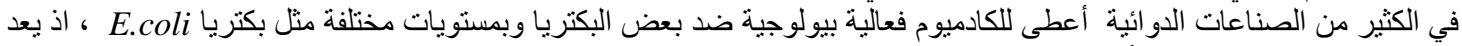

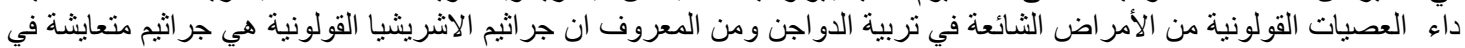

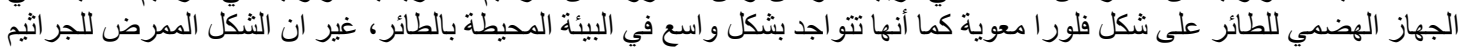

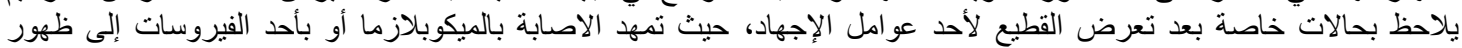

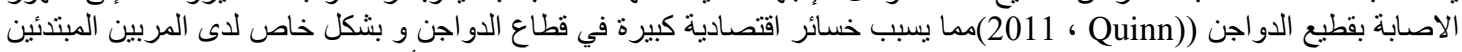

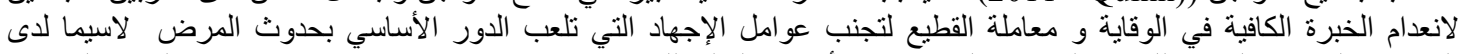

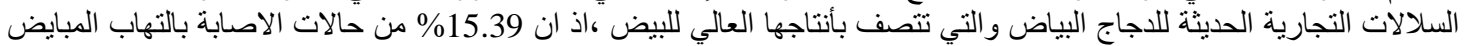

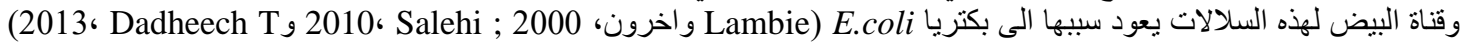

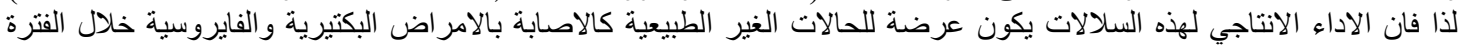

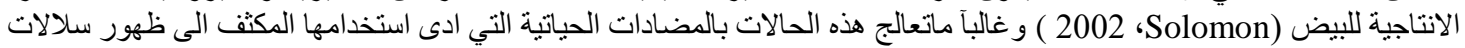

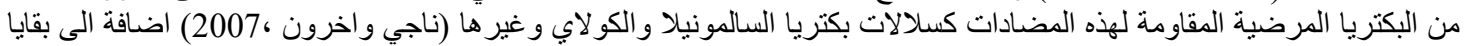

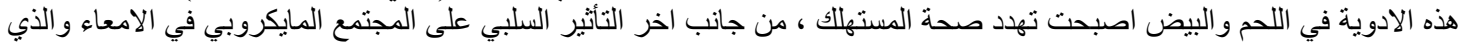

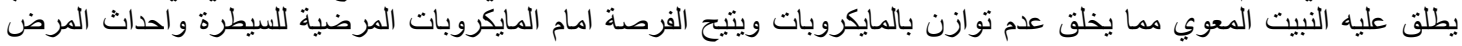

(2011 ، Quinn)

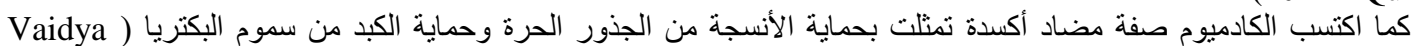

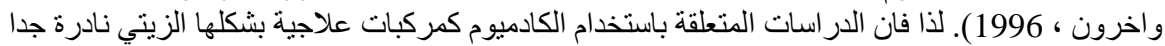

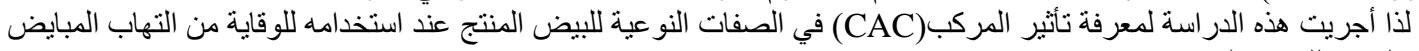

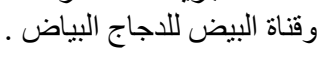




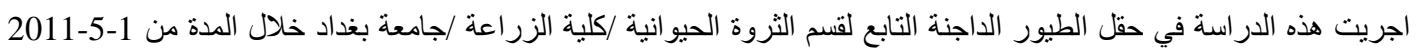

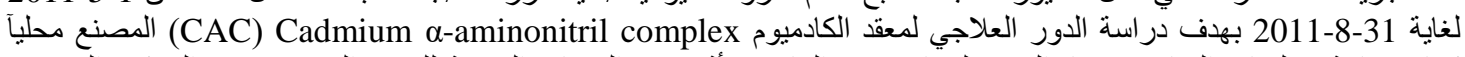

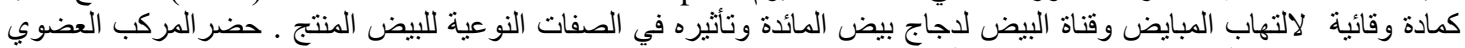

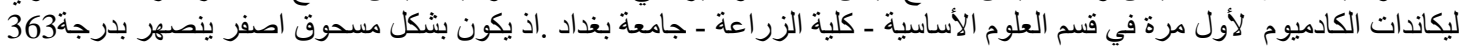

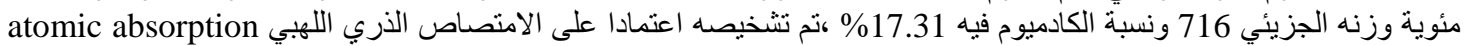

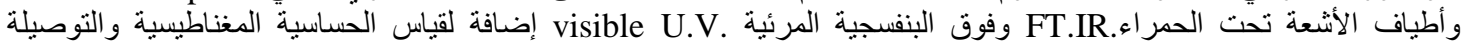

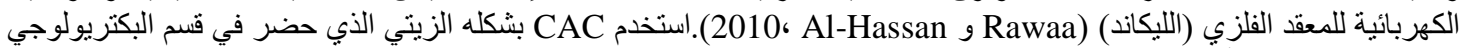

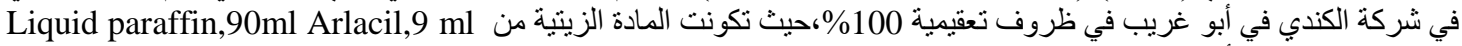
بتر اكيز مختلفة ومزجت باستخدام المازج المغناطيسي

استخدمت 60 دجاجة بياضة سلالة لو همان البني (Lohman brown) بعمر 49 اسبوع تم الحصول عليها من احد الحقول الأهلية

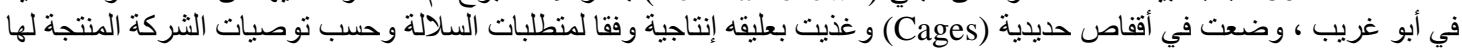

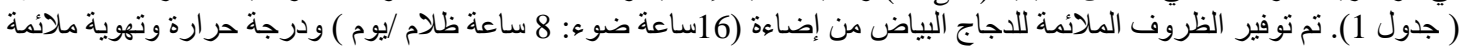

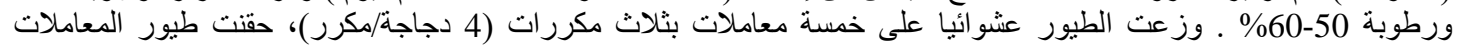

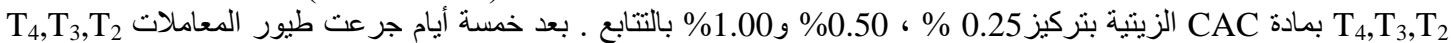

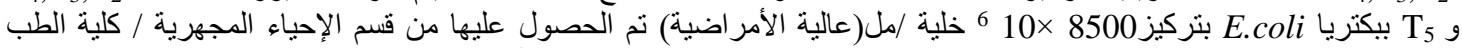

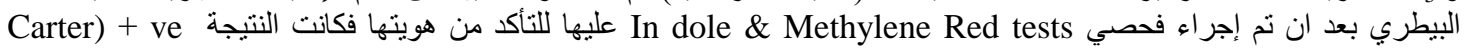

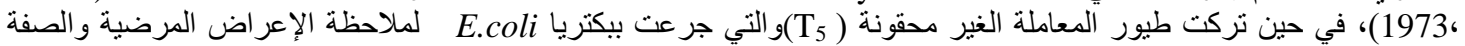

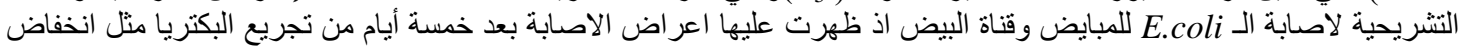

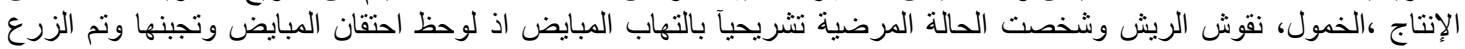

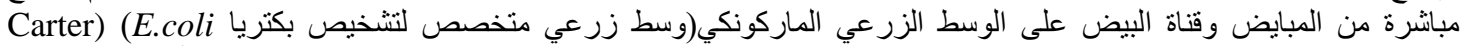

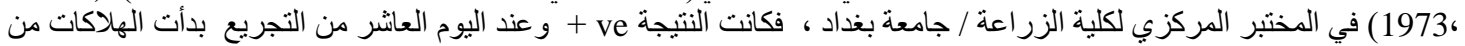

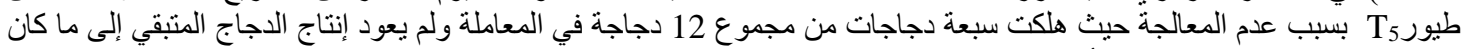

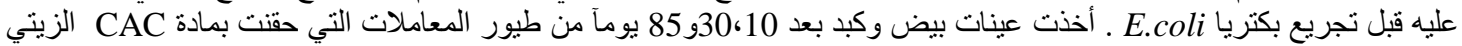

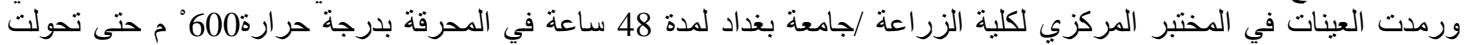

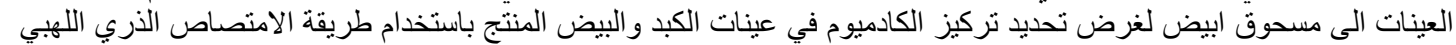

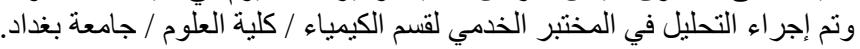

جدول (1). النسب المئوية لمكونات العليقة المستخدمة في التجربة.

\begin{tabular}{|c|c|}
\hline$\%$ & المادة \\
\hline 30.8 & ذرة صفر اء \\
\hline 37.0 & حنطة \\
\hline 18.0 & كسبة فول الصويا (cp\%48) \\
\hline 5.0 & مركز بروتيني(40\%) \\
\hline 0.1 & معادن وفيتامينات \\
\hline 0.3 & زيت الذرة \\
\hline 7.5 & كربونات الكالسيوم \\
\hline 0.3 & ملح \\
\hline 1.0 & فوسفات ثنائي الكالسيوم (18 \\
\hline
\end{tabular}

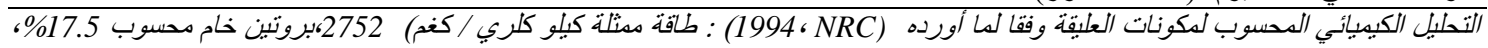

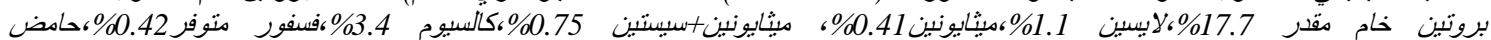
اللبيولينك 1.05\%

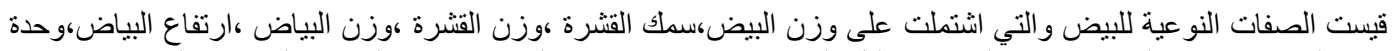

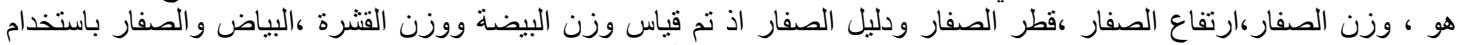

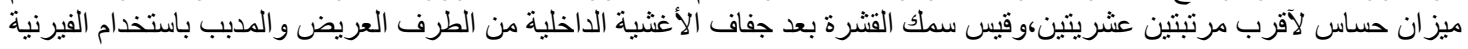

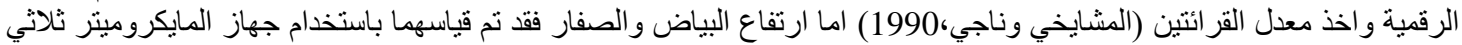

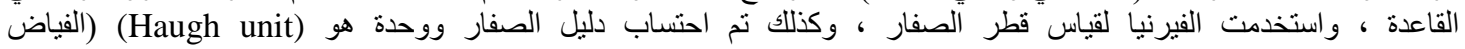

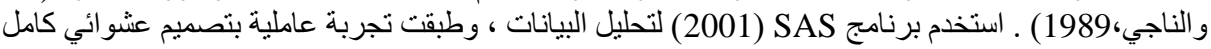
Duncuns Complete Randomized Design(CRD) (1955، Duncan ) Multiple Range ) وبمستوى احتمالية (P<0.01) 
فحص CAC مختبريا كمضاد حياتي كيميائي مصنع بإجر اء فحص الحساسية على زرع بكتيريا لـ E.coli فكانت النتيجة مشجعة

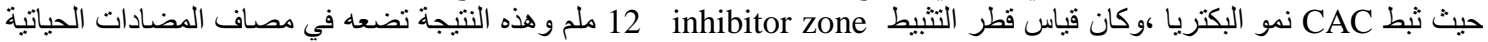
الجيدة جدا مبلئيا.

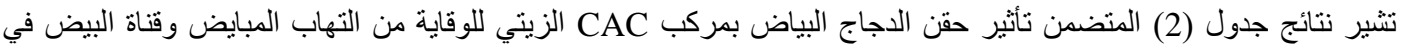

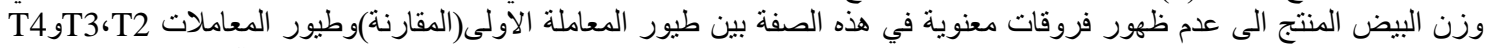

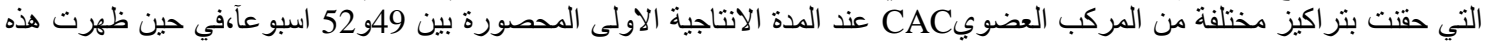

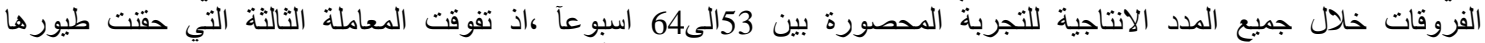

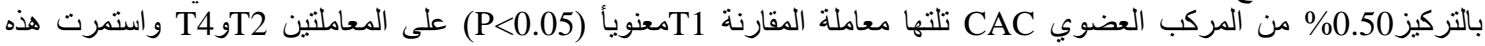

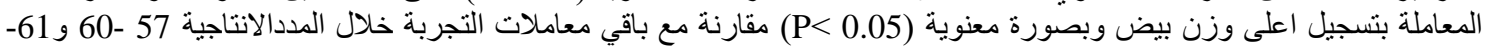
64اسبو عاً و عند حساب المعدل العام للتجربة (49-64) اسبو عاً.

جدول (2). تأثير حقن تراكيز مختلفة من CAC الزيتي في دجاج بيض المائدة في متوسط وزن البيضة(غم)،وزن القثرة(غم) وسمك

\begin{tabular}{|c|c|c|c|c|c|}
\hline & & & & & القشرة \\
\hline \multicolumn{6}{|c|}{ متوسطوزن البيض(غر) للأسابيع الإنتاجية } \\
\hline المعدل العام & $64-61$ & $60-57$ & $56-53$ & $52-49$ & المعاملة \\
\hline $0.4 \mathrm{~b} \pm 59.18$ & $0.64 \mathrm{c} \pm 59.37$ & $0.44 c \pm 59.02$ & $2.19 \mathrm{a} \pm 59.00$ & $59.30 \pm 0.35$ & $\mathrm{~T} 1$ \\
\hline $0.8 \mathrm{~b} \pm 59.63$ & $0.79 b \pm 60.12$ & $0.56 \mathrm{~b} \pm 60.30$ & $2.56 \mathrm{~b} \pm 58.09$ & $59.99 \pm 2.56$ & $\mathrm{~T} 2$ \\
\hline $0.8 \mathrm{a} \pm 61.10$ & $0.60 \mathrm{a} \pm 62.22$ & $0.38 \mathrm{a} \pm 62.11$ & $1.82 \mathrm{a} \pm 60.17$ & $0.20 \pm 59.81$ & T3 \\
\hline $0.5 \mathrm{cb} \pm 58.46$ & $0.49 \mathrm{c} \pm 59.08$ & $1.02 \mathrm{c} \pm 58.43$ & $2.02 b \pm 57.66$ & $58.67 \pm 2.07$ & $\mathrm{~T} 4$ \\
\hline$*$ & * $\quad$ ( & & $*$ & N.S & 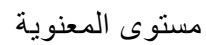 \\
\hline \multicolumn{6}{|c|}{ متوسط وزن قشرة البيض(غم) للاسابيع الانتاجية } \\
\hline المعدل العام & $61-64$ & $60-57$ & $56-53$ & $52-49$ & المعاملة \\
\hline $5.43 \pm 0.14$ & $5.78 \pm 0.09$ & $4.94 \pm 0.14$ & $5.32 \pm 0.12$ & $5.65 \pm 0.14$ & $\mathrm{~T} 1$ \\
\hline $5.39 \pm 0.19$ & $5.39 \pm 0.18$ & $40.68 \pm 0.54$ & $5.74 \pm 0.01$ & $5.73 \pm 0.32$ & $\mathrm{~T} 2$ \\
\hline $5.39 \pm 0.15$ & $5.27 \pm 0.08$ & $5.02 \pm 0.07$ & $5.21 \pm 0.40$ & $6.06 \pm 0.1$ & T3 \\
\hline $5.33 \pm 0.15$ & $5.45 \pm 0.22$ & $4.92 \pm 0.44$ & $5.49 \pm 0.36$ & $5.46 \pm 0.02$ & $\mathrm{~T} 4$ \\
\hline N.S & N.S & N.S & N.S & N.S & 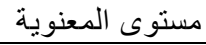 \\
\hline \multicolumn{6}{|c|}{ متوسط سمك قشرة البيض(ملم) للاسابيع الانتاجية } \\
\hline المعدل العام & $64-61$ & $60-57$ & $56-53$ & $52-49$ & المعاملة \\
\hline $0.34 \pm 0.06$ & $0.36 \pm 0.09$ & $0.35 \pm 0.14$ & $0.35 \pm 0.12$ & $0.33 \pm 0.14$ & $\mathrm{~T} 1$ \\
\hline $0.35 \pm 0.006$ & $0.35 \pm 0.07$ & $0.36 \pm 0.02$ & $0.33 \pm 0.01$ & $0.35 \pm 0.01$ & $\mathrm{~T} 2$ \\
\hline $0.35 \pm 0.02$ & $0.35 \pm 0.02$ & $0.36 \pm 0.04$ & $0.31 \pm 0.1$ & $0.35 \pm 0.00$ & T3 \\
\hline $0.36 \pm 0.009$ & $0.37 \pm 0.02$ & $0.36 \pm 0.002$ & $0.35 \pm 0.03$ & $0.35 \pm 0.006$ & $\mathrm{~T} 4$ \\
\hline N.S & N.S & N.S & N.S & N.S & 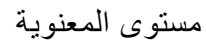 \\
\hline
\end{tabular}

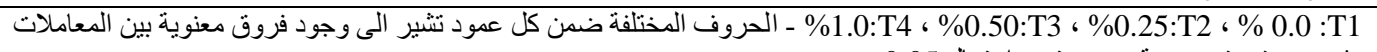

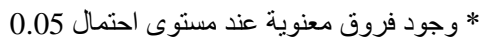

عدم و Nود فروقات معنوية بين متوسطات قيم العمود الو احد. N.S

من الجدول نفسه نلاحظ عدم وجود فروقات معنوية بين المعاملات المختلفة في صفتي وزن وسمك القشرة خلال جميع المراحل

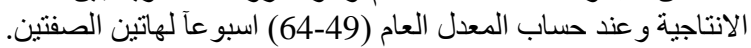

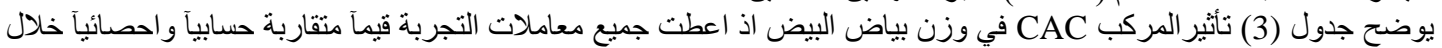

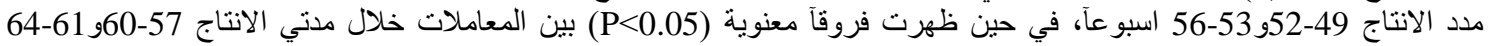

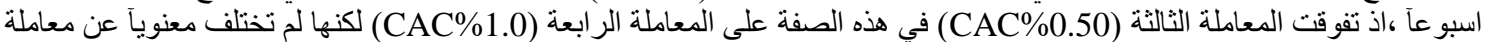

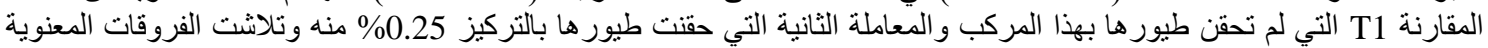

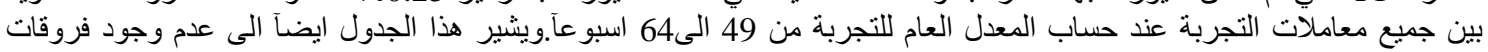

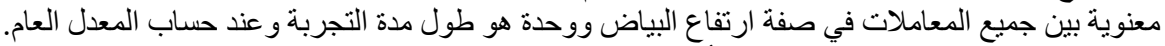

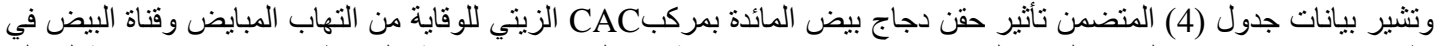

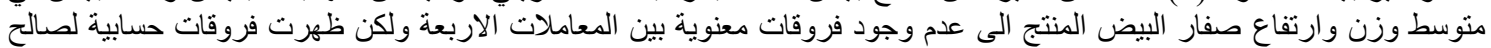

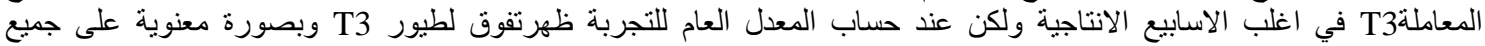

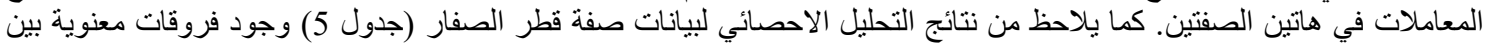

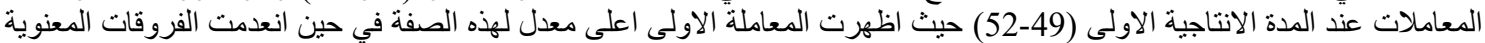

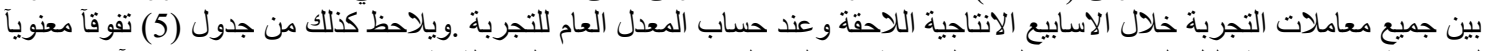

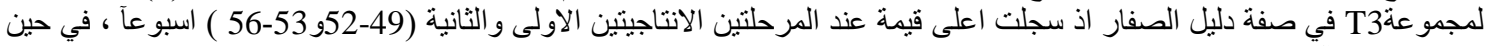

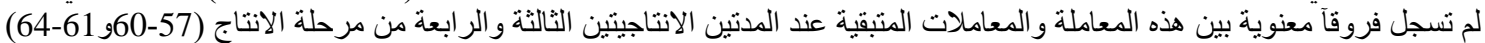

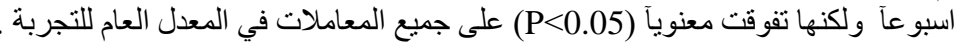


Hammed

جدول (3). ت تأثير حقن تراكيز مختلفة من CAC الزيتي في دجاج بيض المائدة في متوسط وزن البياض (غم)،ارتفاع البياض(ملم) ووحدة هو.

\begin{tabular}{|c|c|c|c|c|c|}
\hline \multicolumn{6}{|c|}{ منوسط وزن البياض(غم) للأسابيع الإنتاجية } \\
\hline المعدل العام & $64-61$ & $60-57$ & $56-53$ & $52-49$ & 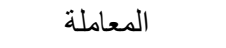 \\
\hline $38.83 \pm 0.58$ & $39.02 \pm 0.40 \mathrm{ab}$ & $39.25 \pm 1.71 \mathrm{ab}$ & $39.35 \pm 1.67$ & $37.69 \pm 0.59$ & $\mathrm{~T} 1$ \\
\hline $38.60 \pm 0.79$ & $38.78 \pm 0.8 \mathrm{ab}$ & $39.17 \pm 1.08 \mathrm{ab}$ & $37.79 \pm 1.95$ & $38.69 \pm 2.79$ & $\mathrm{~T} 2$ \\
\hline $39.58 \pm 0.67$ & $40.73 \pm 0.40 \mathrm{a}$ & $40.92 \pm 1.62 \mathrm{a}$ & $38.99 \pm 2.33$ & $37.68 \pm 1.37$ & $\mathrm{~T} 3$ \\
\hline $37.60 \pm 0.46$ & $37.81 \pm 0.32 \mathrm{~b}$ & $37.35 \pm 0.98 b$ & $37.41 \pm 0.68$ & $37.83 \pm 1.73$ & $\mathrm{~T} 4$ \\
\hline N.S & $*$ & $*$ & N.S & N.S & مستوى المعنوية \\
\hline & & & & \multicolumn{2}{|c|}{ بط ارتفاع البياض (ملم) للاسابيع الانتاجية } \\
\hline المعدل العام & $64-61$ & $60-57$ & $56-53$ & $52-49$ & المعاملة \\
\hline $6.78 \pm 0.14$ & $7.99 \pm 0.09$ & $7.10 \pm 0.14$ & $6.43 \pm 0.17$ & $6.56 \pm 0.41$ & $\mathrm{~T} 1$ \\
\hline $6.36 \pm 0.19$ & $6.26 \pm 0.18$ & $6.52 \pm 0.74$ & $6.41 \pm 0.01$ & $6.25 \pm 0.32$ & $\mathrm{~T} 2$ \\
\hline $6.64 \pm 0.25$ & $6.40 \pm 0.17$ & $6.61 \pm 0.33$ & $7.65 \pm 0.60$ & $5.92 \pm 0.40$ & $\mathrm{~T} 3$ \\
\hline $6.58 \pm 0.15$ & $8.00 \pm 0.09$ & $6.87 \pm 0.32$ & $6.90 \pm 0.36$ & $6.17 \pm 0.33$ & $\mathrm{~T} 4$ \\
\hline N.S & N.S & N.S & N.S & N.S & مستوى المعنوية \\
\hline \multicolumn{6}{|c|}{ متوسط وحدة هو للاسابيع الانتاجية } \\
\hline المعدل العام & 64-61 & $60-57$ & $56-53$ & $52-49$ & المعاملة \\
\hline $86.88 \pm 0.59$ & $87.63 \pm 0.32$ & $87.13 \pm 0.93$ & $86.84 \pm 2.03$ & $85.92 \pm 0.70$ & $\mathrm{~T} 1$ \\
\hline $85.92 \pm 0.85$ & $87.17 \pm 0.45$ & $85.30 \pm 1.15$ & $83.84 \pm 1.17$ & $84.84 \pm 2.02$ & $\mathrm{~T} 2$ \\
\hline $87.73 \pm 0.70$ & $88.63 \pm 0.74$ & $88.71 \pm 0.12$ & $87.82 \pm 2.42$ & $85.74 \pm 1.19$ & $\mathrm{~T} 3$ \\
\hline $85.04 \pm 0.66$ & $86.38 \pm 0.63$ & $86.54 \pm 0.31$ & $84.50 \pm 2.58$ & $86.25 \pm 2.74$ & $\mathrm{~T} 4$ \\
\hline N.S & N.S & N.S & N.S & N.S & مستوى المعنوية \\
\hline
\end{tabular}

جدول (4). تأثير حقن تراكيز مختلفة من CAC الزيتي في دجاج بيض المائدة في متوسط وزن الصفار(غم) وارتفاع الصفار(ملم).

\begin{tabular}{|c|c|c|c|c|c|}
\hline \multicolumn{5}{|c|}{ متوسط وزن الصفار (غم) للأسابيع الإنتاجية } & \multirow[b]{2}{*}{ 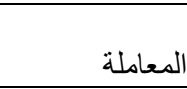 } \\
\hline المعدل العام & $64-61$ & $60-57$ & $56-53$ & $52-49$ & \\
\hline $15.84 \pm 0.29 b$ & $15.83 \pm 0.23$ & $15.82 \pm 0.67$ & $15.73 \pm 0.48$ & $15.99 \pm 0.64$ & $\mathrm{~T} 1$ \\
\hline $15.56 \pm 0.24 b$ & $15.95 \pm 0.13$ & $16.17 \pm 0.04$ & $14.55 \pm 0.52$ & $15.42 \pm 0.45$ & $\mathrm{~T} 2$ \\
\hline $16.12 \pm 0.27 \mathrm{a}$ & $17.22 \pm 0.15$ & $16.17 \pm 1.01$ & $15.97 \pm 0.37$ & $16.08 \pm 0.65$ & T3 \\
\hline $15.53 \pm 0.27 b$ & $15.81 \pm 0.46$ & $16.16 \pm 0.49$ & $14.76 \pm 0.51$ & $15.38 \pm 0.16$ & $\mathrm{~T} 4$ \\
\hline$*$ & N.S & N.S & N.S & N.S & مستوى المعنوية \\
\hline \multicolumn{5}{|c|}{ متوسط ارتفاع الصفار(ملم ) للاسابيع الانتاجية } & \multirow[b]{2}{*}{ 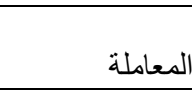 } \\
\hline المعدل العام & $64-61$ & $60-57$ & $56-53$ & $52-49$ & \\
\hline $14.47 \pm 0.18 \mathrm{c}$ & $16.01 \pm 0.16$ & $15.78 \pm 0.27$ & $15.07 \pm 0.17$ & $15.02 \pm 0.40$ & $\mathrm{~T} 1$ \\
\hline $15.54 \pm 0.29 b$ & $15.99 \pm 0.30$ & $15.59 \pm 0.48$ & $15.33 \pm 1.06$ & $15.24 \pm 0.53$ & $\mathrm{~T} 2$ \\
\hline $16.09 \pm 0.13 \mathrm{a}$ & $16.31 \pm 0.25$ & $15.89 \pm 0.13$ & $16.19 \pm 0.05$ & $15.99 \pm 0.3$ & T3 \\
\hline $15.45 \pm 0.23 b$ & $16.28 \pm 0.22$ & $16.02 \pm 0.22$ & $14.98 \pm 0.11$ & $14.51 \pm 0.12$ & $\mathrm{~T} 4$ \\
\hline$*$ & N.S & N.S & N.S & N.S & مستوى المعنوية \\
\hline
\end{tabular}

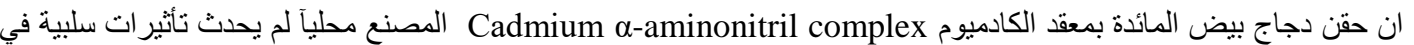

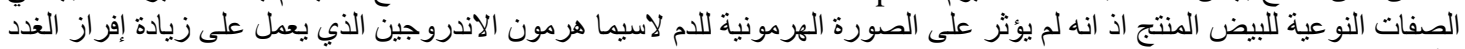

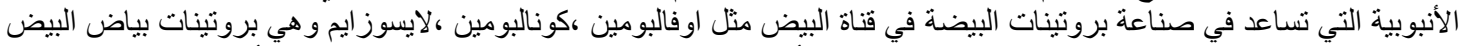

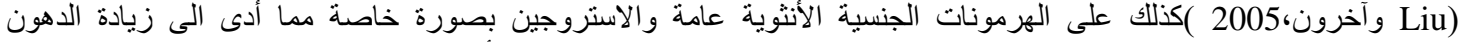

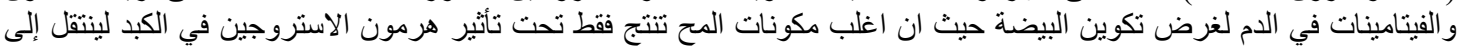

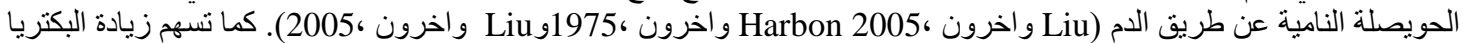

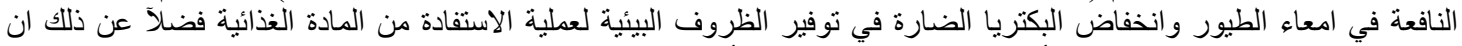

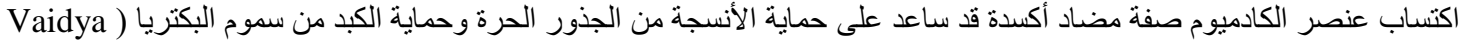

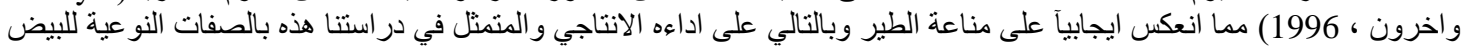
المنتج من قبل الدجاج المعالج بمعقد الكادميوم. 
Egyptian J. Nutrition and Feeds (2015)

جدول (5). تأثير حقن تراكيز مختلفة من CAC الزيتي في دجاج بيض المائدة في متوسط قطر الصفار(ملم) ودليل الصفار.

\begin{tabular}{|c|c|c|c|c|c|}
\hline \multicolumn{5}{|c|}{ متوسط قطر الصفار (ملم) للاسابيع الانتاجية } & \multirow[b]{2}{*}{ 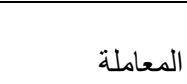 } \\
\hline المعدل العام & $64-61$ & $60-57$ & $56-53$ & $52-49$ & \\
\hline $36.10 \pm 0.48$ & $35.85 \pm 0.55$ & $36.57 \pm 1.26$ & $35.51 \pm 0.93$ & $36.44 \pm 1.41 \mathrm{a}$ & $\mathrm{T} 1$ \\
\hline $35.72 \pm 0.43$ & $35.99 \pm 0.40$ & $36.32 \pm 0.38$ & $34.88 \pm 0.31$ & $35.68 \pm 1.26 \mathrm{~b}$ & $\mathrm{~T} 2$ \\
\hline $35.26 \pm 0.46$ & $36.57 \pm 0.66$ & $36.09 \pm 0.38$ & $34.94 \pm 0.40$ & $33.42 \pm 1.05 \mathrm{c}$ & T3 \\
\hline $35.36 \pm 0.43$ & $36.51 \pm 0.46$ & $36.01 \pm 0.21$ & $34.40 \pm 0.63$ & $34.50 \pm 0.91 \mathrm{c}$ & $\mathrm{T} 4$ \\
\hline N.S & N.S & N.S & N.S & $*$ & مستوى المعنوية \\
\hline \multicolumn{6}{|c|}{ متوسط دليل الصفار للاسابيع الانتاجية } \\
\hline 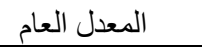 & $64-61$ & $60-57$ & $56-53$ & $52-49$ & 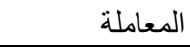 \\
\hline $0.43 \pm 0.009 \mathrm{~b}$ & $0.44 \pm 0.01$ & $0.43 \pm 0.02$ & $0.43 \pm 0.02 \mathrm{~b}$ & $0.41 \pm 0.02 \mathrm{~b}$ & T1 \\
\hline $0.43 \pm 0.08 \mathrm{~b}$ & $0.44 \pm 0.005$ & $0.43 \pm 0.009$ & $0.43 \pm 0.03 \mathrm{~b}$ & $0.43 \pm 0.02 b$ & $\mathrm{~T} 2$ \\
\hline $0.46 \pm 0.005 \mathrm{a}$ & $0.45 \pm 0.02$ & $0.44 \pm 0.004$ & $0.46 \pm 0.05 \mathrm{a}$ & $0.48 \pm 0.01 \mathrm{a}$ & T3 \\
\hline $0.43 \pm 0.005 b$ & $0.45 \pm 0.008$ & $0.44 \pm 0.003$ & $0.43 \pm 0.01 \mathrm{~b}$ & $0.41 \pm 0.01 \mathrm{~b}$ & $\mathrm{~T} 4$ \\
\hline$*$ & N.S & N.S & $*$ & $*$ & مستوى المعنوية \\
\hline
\end{tabular}

جدول (6). تركيز الكادميوم في نماذج الكبا والبيض المرمدين للاجاج البياض المأخوذة من T4،T3، T2.

\begin{tabular}{|c|c|c|c|}
\hline تركيز الكادمييوم في & عدد النماذج & لمدد التي أخذت فيها العينة بعد عملية & العينة \\
\hline-0.027 & 18 & 10 & البيض \\
\hline 0.006 & 18 & 30 & البيض \\
\hline 0.006 & 18 & 85 & البيض \\
\hline-0.027 & 18 & 30 & الكبد \\
\hline-0.029 & 18 & 65 & الكبد \\
\hline
\end{tabular}

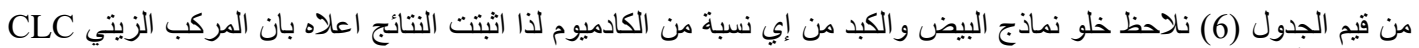

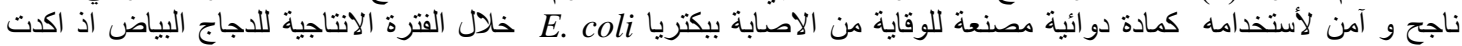

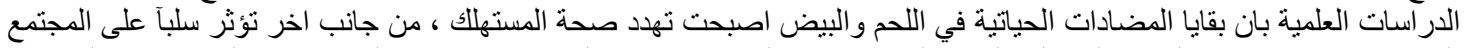

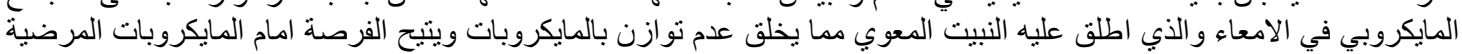

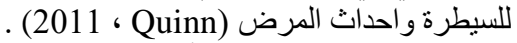

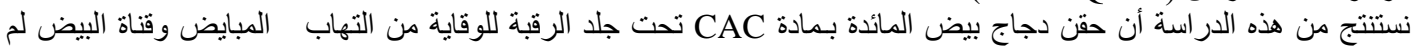

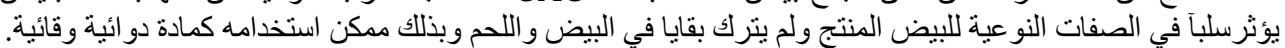

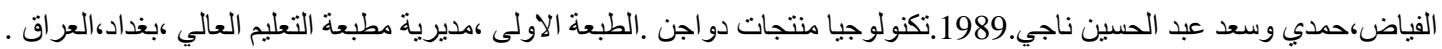

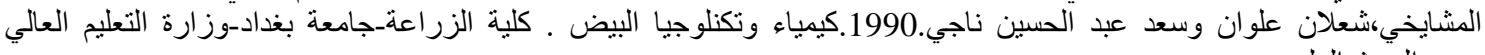

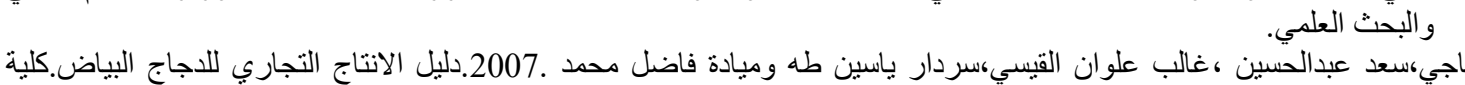
الز الزر اعة-جامعة بغداد.

Ahmed, H. and A.M. jamal (2009). Comparative studies of the determina-tion of divalent Cadmium, Lead and Copper in the boiling medicinal herbs by stripping Voltammetry and by atomic absorption spectrometry. American J. of Applied Sciences, 6(4):594-600.

Carter, G.R. (1973). Diagnositic procedures of veterinary microbiology $2^{\text {nd }}$ ed. Clinical microbiology Lab., Depart. Of microb.and public health, Med. State univ. East Lansing, Mischign spring field, Illinois, USA.p:53.

Dadheech, T. and R. Vyas (2013). Clinical and gross-patholigical diagnosis of infectious bacterial diseases of layer chickens in Ajmer region of Rajasthan .Res J. Pharm. Biol. Chem. Sci., 4:15361548.

Duncan, D.B. (1955). Multiple range and multiple f. test biometrics, 11:1-42.

Harbon, J.B.; T.J. Marby and H.Marby (1975).The flavonoides, Chapman and Hall, London. 


\section{Hammed}

Hidetoyo, T.; K. Takagawa; Y. Arai; K. Wakaki; Y. Sum and K. Takaya (2002). Histological staining of cadmium with 2- (8-quindylazo) 4,5diphenylimidazole (QAI) in the kidneys of rats per orally to cadmium. J. Occup. Health, 44:60-62.

Jeyaprakash, K. and P.Chinnaswamy (2005). Effect of Spirulina and Liv.52 on Cadmium induced Toxicity in Albino Rats. Indian J. of Experi.Biol.43, p773-781.

Khandelwal, S.; D. Kochru and S.K. Tandon (1987). Influence of metal chelators on metallo enzymes Toxical Lett.37 p.:213.

Liu, M.J.; Z. Wong and R.N. Wu (2005). Diosgenin induced cell cycle arres and in apoptosis human leukemia K562 cell with distribution of Ca 2t homeostasis. Cancer chemo the rpharm., 55(1):79-90.

Lambie NI, Ngeleka M, Brown G, Ryan j.2000. Retrospective study on Escherichia coli infection in broilers subjected to postmortem examination and antibiotic resistance of isolated in Trinidad. Avian Disease, Jan-Mar. 44(1):60-155.

NRC, National Research Council.1994.Nutrient requirements of poultry.9th ed., National Academic Press, Washington, DC. USA.pp.120.

Nordberg, G.F.; T. Kjellstorm and G.E. Noedberg (1985). Cadmium and Health toxicological and Epidemiological appraisal, (CRC-Press, Boca Raton, USA), 1:168.

Pirarat, P.; P. Chotipong and P. Singhasenee (2008). Toxicity of Cadmium on Tilapia (Oreochromis niloticus) Spleen. The $5^{\text {th }}$ Congress of FAVA, p: 143-144.

Quinn, P.J.; B.K. Markey; F.C. Leonard; E.S. Fitzpatrick; S. Fanning and P. Hartigen. J. Veterinary microbiology and microbial disease. $2^{\text {nd }}$ ed .USA: John Wiley and Sons: 2011.pp.263-286.

Rawaa, A.M. and Kh. Abd- Al-Hassan (2010).Synthesis and Characterization of New Metal Complexes of $\alpha$-Aminonitrile Derived from P- Toluidine and Aromatic Aldehyde . J. Baghdad Sci., 7(3); 1214122.

Salehi, M. and R. Ghanbarpour (2010). Characterization of Escherichia coli isolates from commercial layer hens with salpinbitis. Am.J.Anim.Vet Sci,. 5:208-214.

SAS (2001). SAS/STAT Users Guide for Personal Computers Release b.12 SAS. Institute, Inc. Cary. NC. USA.

Solomon, S.E. (2002). The oviduct in chaos. Worlds Poult. Sci. J., (58):41-48.

Vaidya, A.B.; S.M. Sirsat; J.C. Doshi and D.S. Antarkar (1996). Selected medical plants and formulation as hepatobiliary drugs. Indian clin.pharmacol.Ther., 17:p.7 


\title{
THE EFFECT OF INJECTION LAYER HENS WITH CADMIUM $\alpha-$ AMINONITRIL COMPLEX ON EGG QUALITY TRAITS.
}

\author{
Sanaa. A. Al- Hammed \\ Animal Resource Department -College of Agriculture-University of Baghdad-Iraq.
}

\section{SUMMARY}

This study was carried out at the poultry farm of Animal Resources Dep./ college of Agriculture / University of Baghdad during the period from 1-5-2011 to 31-8-2011, to study the effect of a new local oily injectable cadmium complex (CAC) [Di nitrate- bis \{p-methyl anilino phenyl aceto nitrite $\}$ cadmium (II)]-2H2O with molecular formula [Cd (HL) 2(NO3)2] - 2H2O in layer hens to control ovaritis and salpingitis and its effect on egg quality traits. 60 healthy Lohmann Brown laying hens 49 weeks age were used in this study. The hens were randomly distributed into five treatments reach treatment included three replicates (4 hens / replicate).

The CAC was injected subcutaneously in layer hens neck at different levels where T1: 0.0\% (uninjected, uninfected as normal control), T2: $0.25 \%$ ، T3: $0.50 \%$ and T4: 1.00\%. After five days T2, T3,T4 and T5 were orally administrated with E.coli by drinking water at concentration of $8500 \times 10^{6} \mathrm{cells} / \mathrm{ml}$ (highly pathogenic) as causative agent of Salpengitis and Ovaritis, while T5 assumed as uninjected treatment but treated with E.coli. The results revealed that the treated birds (T2, T3, and T4) showed a resistance against E.coli infection. However, birds injected with $0.50 \% \mathrm{CAC}(\mathrm{T} 3)$ showed superiority in egg weight as compared to control group T1,T2 and T4, respectively, during the production weeks 57-60 and 61-64 as well as at the total mean of the experiment $49-64$ wks.T3 also showed superiority in albumen weight during these two production periods as compared to T4, while the mentioned treatment (T3) showed superiority over all other experiment groups in weight and height yolk at the total mean of the experiment , and in yolk index during the two production periods 49-52 and 53-56 wks. However, no significant differences among different treatments in shell weight, shell thickness, albumin height and Haugh unit. The results also showed that the liver and egg samples of treated birds were free from cd (11) ion residuals.

The obtained results refered to the safety use of CAC as a synthetic chemical prophylactic agent against E.coli during the production period of layer hens.

Keyword: layer, egg quality traits, E.coli. Cadmium aminonitrile complex. 